\title{
Polydimethylsiloxane (PDMS)-Based Flexible Resistive Strain Sensors for Wearable Applications
}

\author{
Jing Chen ${ }^{1,2}$, Jiahong Zheng ${ }^{1}$, Qinwu Gao ${ }^{2}$, Jinjie Zhang ${ }^{2}$, Jinyong Zhang ${ }^{2}$, \\ Olatunji Mumini Omisore ${ }^{2}$, Lei Wang ${ }^{2, *(D)}$ and Hui Li ${ }^{2, *}$ \\ 1 School of Mechanical and Electrical Engineering, Shaanxi University of Science \& Technology, Xi'an 710021, \\ China; jc@siat.ac.cn (J.C.); jhz@sust.edu.cn (J.Z.) \\ 2 Shenzhen Institutes of Advanced Technology, Chinese Academy of Sciences, Shenzhen 518055, China; \\ qwg@siat.ac.cn (Q.G.); jjz@siat.ac.cn (J.Z.); jy.zhang@siat.ac.cn (J.Z.); omisore@siat.ac.cn (O.M.O.) \\ * Correspondence: wang.lei@siat.ac.cn (L.W.); hui.li1@siat.ac.cn (H.L.); Tel.: +86-0755-8639-2298 (L.W. \& H.L.)
}

Received: 10 January 2018; Accepted: 12 February 2018; Published: 28 February 2018

\begin{abstract}
There is growing attention and rapid development on flexible electronic devices with electronic materials and sensing technology innovations. In particular, strain sensors with high elasticity and stretchability are needed for several potential applications including human entertainment technology, human-machine interface, personal healthcare, and sports performance monitoring, etc. This article presents recent advancements in the development of polydimethylsiloxane (PDMS)-based flexible resistive strain sensors for wearable applications. First of all, the article shows that PDMS-based stretchable resistive strain sensors are successfully fabricated by different methods, such as the filtration method, printing technology, micromolding method, coating techniques, and liquid phase mixing. Next, strain sensing performances including stretchability, gauge factor, linearity, and durability are comprehensively demonstrated and compared. Finally, potential applications of PDMS-based flexible resistive strain sensors are also discussed. This review indicates that the era of wearable intelligent electronic systems has arrived.
\end{abstract}

Keywords: wearable electronics; strain sensors; flexible substrate; PDMS

\section{Introduction}

Wearable electronic technology integrates electronic devices into clothing, accessories, human skin, and is even implanted in vivo and realizes measurements of body sensing, data storage, and mobile computing [1-9]. The front-end component of wearable systems is a wide variety of wearable sensors, which can be used to measure all kinds of physiological parameters, such as body temperature $[10,11]$, myoelectricity [12], heart rate [13], and blood glucose [14]. They are also used for human motion detection, such as acceleration [15], muscle ductility [6,16], and foot pressure [17,18]; as well as environment monitoring, for example, position coordinates [19], temperature [20,21], humidity [22,23], and atmospheric pressure [24]. Different from other functional wearable sensors, strain sensors are attached to the joint or even directly mounted on the muscle for human motion inspection [25-29]. They must be capable of not only bending to conform to the topography of the skin but also stretching during natural body activities. Recently, some wearable strain sensors have been proposed to monitor the bending/unbending state of a joint [30] and to collect physiological signals of a volunteer in relaxation and after exercise [31,32].

Wearable strain sensors typically consist of a sensitive element and a supporting mechanism, and their flexibility is deeply influenced by the sensing mechanism and the substrate material. Compared to other transduction techniques, including capacitive [33], optoelectric [34], and piezoelectric [35], the resistive transduction technique is utilized as the primary choice of sensing mechanism owing to its high spatial resolution, relatively simple input/output control system, and low noise susceptibility [36,37]. 
Polydimethylsiloxane (PDMS) has been a common material for microfabrication for decades. Thanks to its stable chemical properties, biological compatibility, transparency, and good thermal stability, and especially its adhesion and non-adhesion areas that are clearly visible under UV light and can be easily adhered to the surface of electronic materials [38-40], PDMS is widely used for microfluidic chips [41], micropumps [42], and electronic skin [43,44]. Particularly, PDMS as a flexible substrate for wearable resistive strain sensors has been developed because of its broad applications in entertainment technology, human-machine interfaces, personal healthcare, and sports performance monitoring, etc. [45-47].

In this review, we primarily focus on microfabrication materials and methods, strain sensing performances, and the applications of PDMS-based flexible resistive strain sensors. The article is organized as follows: first, various component materials and the fabrication methods are described in detail. Second, we compare and summarize the performance parameters between different PDMS-based flexible resistive strain sensors in terms of stretchability, gauge factor, linearity, and durability. Third, we emphasize the applications of PDMS-based flexible resistive strain sensors in some related fields. The last section provides conclusions and the challenges in future research.

\section{Materials and Fabrication Methods}

Flexibility and extensibility of materials are key to fabricating wearable resistive strain sensors. The substrate functions as the flexible support structure of the wearable resistive strain sensors; the properties of the substrate materials directly determine the sensor's elastic performance. Many commercialized polymers can be used to construct flexible substrates for wearable resistive strain sensors; these include polyethylene terephthalate (PET) [48], polyimide (PI) [49], polyethylene (PE) [50], polyurethane (PU) [51], Ecoflex [52], and PDMS [53]. Among them, PDMS is the most widely used material for flexible substrate fabrication because of its excellent comprehensive performance. Another important consideration for flexible sensors is the appropriate active material as the sensing element. The function of the sensing element is to convert external mechanical stimuli into electrical signals. To date, PDMS-based flexible resistive strain sensors have been fabricated by using conducting materials (e.g., EGaIn, Ti/Au, silver nanowires (AgNWs), and Ag nanoparticles (Ag NPs)) [53-56], carbon materials (e.g., graphene, graphene oxide (rGO), carbon nanotubes (CNTs), and carbon black (CB)) [4,57-59], and their hybrid micro/nano-composite [60].

As the material science and microelectromechanical (MEMS) technology has developed, many methods have become available for fabricating flexible sensors. In this section, the typical fabrication methods of PDMS-based flexible resistive strain sensors are summarized as follows. The filtration method [61,62] is a powerful tool for obtaining uniform composite films with filter membranes; the films can be used as a sensing element converting the mechanical deformation into electrical signals. Kyun Kun Kim et al. demonstrated a multidimensional strain sensor composed of prestrained AgNW percolation network lays, which was obtained by the filtration of prestrained AgNW solution and transferred to a PDMS substrate [54]. Chaoyi Yan et al. reported highly stretchable strain nanopapers by mixing crumpled graphene with nanocellulose through vacuum filtration to obtain uniform composite films, and then embedding in a PDMS matrix (Figure 1a) [63]. Printing technology [64,65] involves printing the composite through a deposition nozzle with directly pattern electrodes onto a PDMS substrate as the resistive sensing element. A stretchable electronic was fabricated by J. William Boley et al. using a direct writing approach where the liquid metal EGaIn was injected into a PDMS substrate through a blunt syringe needle [66]. Hai-liang Yan et al. applied a coaxial printing method to embed EGaIn into the PDMS and developed a stretchable and conductive cable for monitoring the frequency and amplitude of a curved object (Figure 1b) [67]. Jaehwan Lee et al. reported a new type of flexible strain sensor that can detect both tensile and compressive strains, using single-step direct transfer patterning to transfer Ag NPs-ink from the donor substrate to the microstructured PDMS [47]. The micromolding method [45,58,68-70] employs micromolds to fabricate electrodes or flexible substrates that are especially suitable for irregular microstructures. 
Nanshu Lu et al. demonstrated highly sensitive skin-mountable strain gauges packaged in the substrate of PDMS with carbon black-doped PDMS as straight resistors, and joined by serpentine-shaped interconnects made with carbon-nanotube-doped PDMS through a repeated photoresist pattern and rinse [58]. A piezoresistive sensor-integrated PDMS cantilever was proposed by Dong-Su Kim et al. The micropatterned PDMS cantilever was prepared by patterning photoresist film on $\mathrm{Si}$ wafer; the sensing element (Ti/Au layers) was integrated onto the PDMS cantilever through photolithography and metal etching processes (Figure 1c) [53]. Coating techniques [71-74] use deposition or evaporation methods to make sensing material uniformly grow on the surface of the polymer layers. Xiao Li et al. demonstrated highly stretchable sensitive strain sensors utilizing graphene woven fabrics (GWFs) for strain sensing. GWFs were prepared from chemical vapor deposition (CVD) with methane, then integrated with a rectangular-shaped backing structure made of PDMS [73]. M. Bulut Coskun et al. used a temporary vinyl mask and fabricated an ultrasensitive flexible strain sensor by selectively depositing liquid graphene oxide dispersion on PDMS substrates (Figure 1d) [57]. Liquid phase mixing $[58,69,75,76]$ is prepared by mixing different materials to give the mixed composite stretchable and conductive properties. Heun Park et al. reported a conductive nanocomposite by mixing a vinyl alcohol/MWCNT/PSS solution (Clevios PH 1000 from Heraeus). A skin-integrated transparent strain sensor was fabricated by spin coating this conductive nanocomposite onto a PDMS substrate [77]. Jeong-Ho Kong et al. obtained a conductive carbon composite by dispersing conductive carbon black nanoparticles into a PDMS matrix, which was used to fabricate elastic strain sensors by selectively patterning along micro-bumps on insulative PDMS substrates with the conductive carbon/PDMS composites (Figure 1e) [75]. 
a)

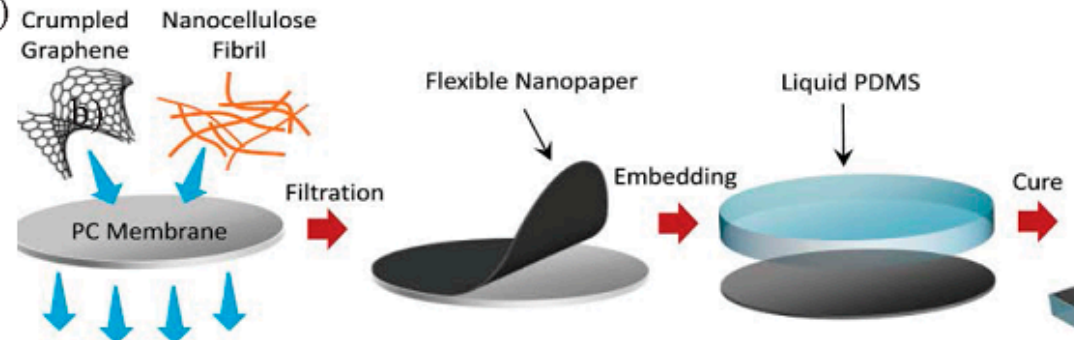

Stretchable Nanopaper

b)

Vacuum Filtration

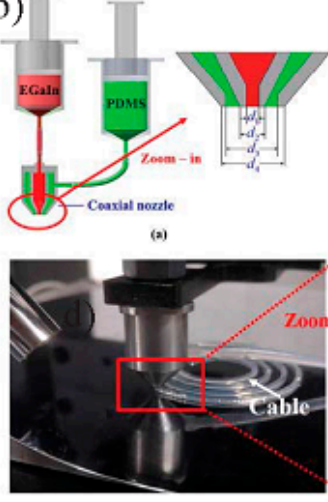

d)

(a)

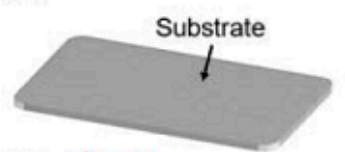

(b)

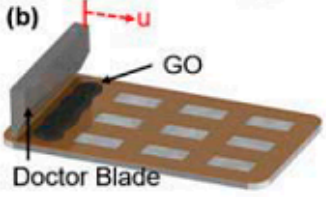

(c) Vinyl masking tape

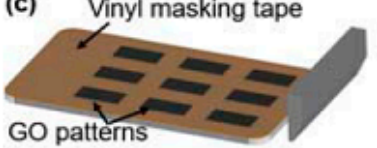

(d)

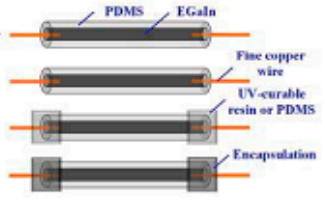

(b)
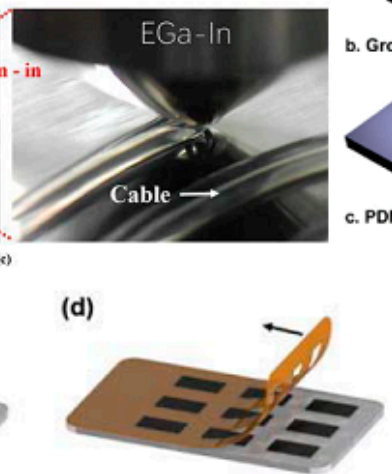

(e)

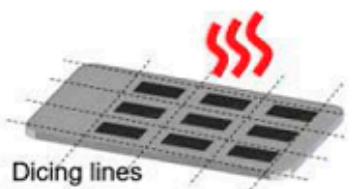

(f) c)

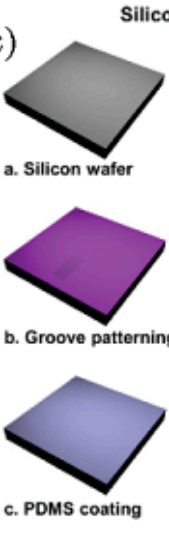

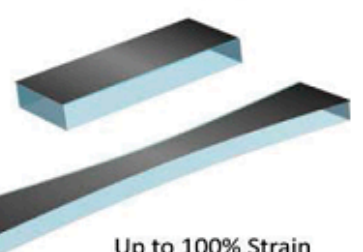

Up to $100 \%$ Strain licon part

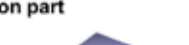

Glass part

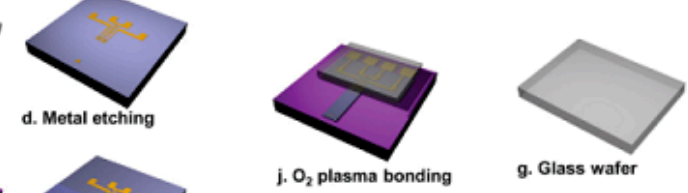

e)
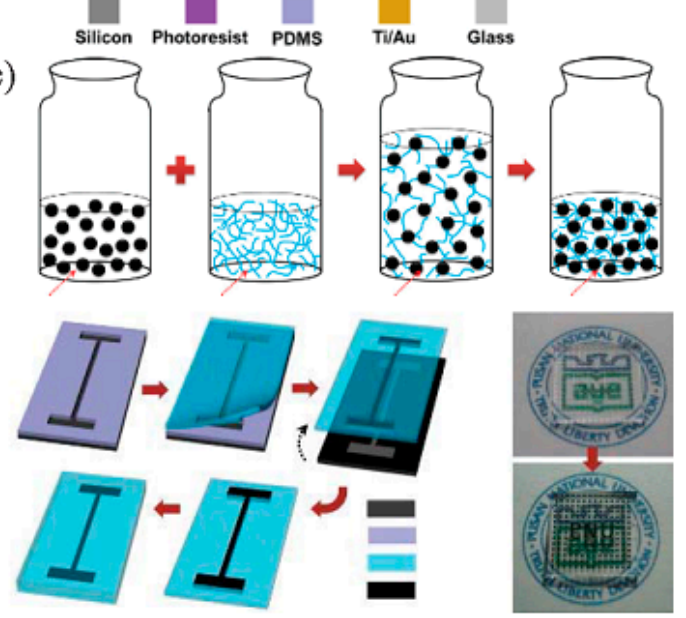

Figure 1. Fabrication methods of resistive-type strain sensors based on polydimethylsiloxane (PDMS) flexible substrates. (a) Schematic illustrations of the fabrication processes for stretchable graphene nanopapers. Flexible nanopapers were first fabricated and then embedded in a PDMS matrix to obtain stretchable nanopapers. Reproduced with permission from [63], Copyright John Wiley and Sons, 2013; (b) The coaxial conductive cable design with EGaIn as the core and PDMS as the shell can directly write for strain sensor. Reprinted with permission from [67], Copyright AIP Publishing, 2016; (c) Strain sensor integrated with PDMS cantilever and metal wires. Reprinted with permission from [53], Copyright Elsevier, 2017; (d) Flexible strain sensor were developed through the combination of shear alignment of a graphene oxide dispersion and patterning of multiple rectangular features on PDMS substrate (Reprinted with permission from [57], Copyright American Chemical Society, 2016; (e) Mixed carbon black nanoparticles with liquid PDMS and micropatterning relies on single-step contact transfer printing. Reprinted from permission with [75], Copyright Elsevier, 2014.

\section{Performance Parameters}

PDMS-based flexible resistive strain sensors are supposed to detect the bending/unbending and stretching/relaxing state of joints, as well as human physiological activities. They respond to 
mechanical deformations by changing electrical resistance properties. Although traditional strain sensors are easy and low cost to fabricate, they have obvious drawbacks such as poor stretchability, low resolution, and not suitable for wearable applications [73]. In many practical applications, it is demanded that wearable strain sensors have high-performance characteristics including stretchability, high gauge factors, linearity, and durability. These parameters are crucial for flexible strain sensors for large deformations, responding sensitivity, frequent loading/ unloading, and wearable time.

\subsection{Stretchability}

Traditional silicon electronics for sensing are rigid and brittle. Large deformations lead to structural damage and performance failure. However, the skin accommodates the movements of the body by bending and stretching itself. Wearable strain sensors must be stretchable to suit body activities. PDMS-based flexible resistive strain sensors have different stretchabilities that depend on their microstructure and sensing component materials. Graphene/PDMS composite strain sensors typically exhibit low stretchability; their measured structural strain is within a range of $0-10 \%$ due to the lack of robust percolating networks of the low aspect ratio nanomaterials $[71,73,74]$. The stretchability is considerably improved to $60-100 \%$ by using silver nanowire networks/PDMS [68], carbon black nanoparticles/PDMS [75], and EGaIn/PDMS [66] as the active sensing material of the flexible strain sensors. Some researchers have taken advantage of carbon nanotube (CNT) films with highly homogeneous microcrack propagation and lateral interconnection; highly stretchable PDMS-based strain sensors have been fabricated and are capable of measuring strains up to $280 \%$ [46]. PDMS-based flexible resistive strain sensors with different stretchabilities can detect different types of human motion, including breathing, speech, blinks, pulses, and bending/unbending of joints.

\subsection{Gauge Factors}

The slope of the resistance-strain curve is defined as the gauge factor (GF) to reflect the sensitivity of a strain sensor. The greater the GF of a sensor, the easier it detects small deformations. The liquid metal EGaIn/PDMS strain sensors typically exhibit a gauge factor within the range of 2-20 [66]; the GFs are controlled by geometry deformation of the liquid metal. The AgNW/PDMS [68] and carbon black nanoparticle/PDMS [75]-based sensors have even smaller gauge factors of 2-10 (Figure 2a). The graphene oxide/PDMS strain sensor exhibits a high GF in the range of 137-261 [57]; a high GF has been achieved by multilayered graphene configurations in which the contacting graphene flakes form an electrical network. Super-sensitive strain sensors have been reported by using graphene woven fabrics (GWF)/PDMS [73] (Figure 2b). The gauge factors of GWF/PDMS sensors have been calculated to be 103 under 2-6\% strains; on the other hand, the gauge factors increase to 106 when under higher strains $(>7 \%)$. The relative change in resistance under strain grows exponentially due to the development of cracks and the dimensional change in the GWF networks. The graphene/PDMS composite strain sensors show GFs of 3.9-233, which can be controlled by the graphene concentrations [74]. In general, for the GF, a higher value is better. However, large GFs can influence the precision of the measuring results because it amplifies noise signals. Therefore, highly sensitive strain sensors must consider the noise-signal ratio. 

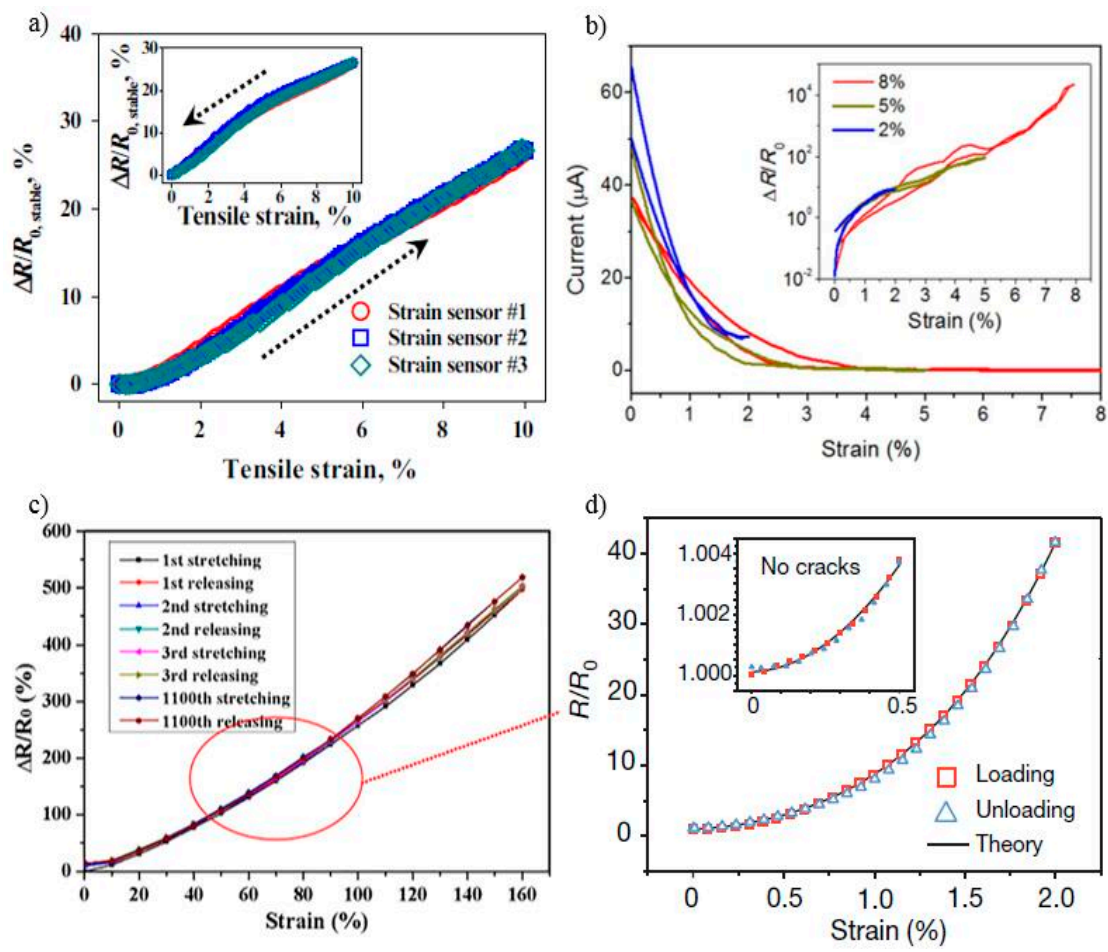

Figure 2. (a) Resistance change ratios obtained from three identical conductive PDMS strain sensors with a maximum strain of $10 \%$ after stabilization. Reprinted with permission from [75], Copyright Elsevier, 2014; (b) The graphene-on-polymer strain sensors show high gauge factors under electrical resistance of graphene woven fabrics, which increases exponentially with tensile strain. Reprinted with permission from [73], Copyright Springer Nature, 2012); (c) Strain sensing capability of stretchable EGaIn/PDMS composite strain sensor with cycles of stretching/releasing. Reprinted with permission from with [67], Copyright AIP Publishing, 2016; (d) Resistance of sensors based on nanoscale crack junctions at low loading-unloading rate compared with the theoretical fit. Reprinted with permission from [78], Copyright Springer Nature, 2014.

\subsection{Linearity}

Under certain conditions, the percentage of maximum deviations between the calibration curve and the fitting line with full-scale output is called the linearity. Linearity is an important performance parameter because the linearity of sensors makes the signals accurate and is very beneficial in the follow-up processing of the output signal. Nonlinearity causes a large deviation between the output signal and the measurement parameter, which results in a complicated calibration process and signal distortion. Carbon black nanoparticle/PDMS composite-based strain sensors typically exhibit linearity and little deviation upon both stretching and releasing with low sensitivity (GFs $\approx 5.5$ ) (Figure 2a). EGaIn/PDMS-embedded structure based strain sensors typically exhibit linearity and stretchability during thousands of cycles of stretching/releasing but still with low sensitivity $(\mathrm{GFs} \approx 3.2)$ (Figure 2c) [67]. Graphene-on-polymer strain sensors and nanoscale crack sensors show excellent sensitivity (GFs $>103$ ) but nonlinearity and low stretchability $(\varepsilon<10 \%$ ) (Figure 2b,d) [73,78]. The main reason for nonlinearity in the resistance response curve is the non-uniform deformation of the sensing element layer on PDMS substrates. A strain sensor responds to the applied strain with nonlinearity when it undergoes heterogeneous deformation. Highly sensitive strain sensors typically respond to the applied strain with nonlinearity and low stretchability because they are always non-uniform deformation. Therefore, developing strain sensors with high linearity, stretchability, and sensitivity is still a grand challenge. 


\subsection{Durability}

Durability represents the ability of the strain sensor to recover its original properties during the reciprocating deformation. Durability is more important for wearable strain sensors in practical applications due to the inevitability of large, complex, and dynamic strains. AgNW/PDMS percolation network strain sensors show long-term reliability during thousands of dynamic cyclic strains $(10 \%)$ with negligible change in the resistance profile (Figure 3a) [54]. A two-layered flexible strain sensor shows excellent dynamic bending stability with the resistance decreasing slightly after 2400 bending cycles (maximum bending angle: $90^{\circ}$ ) (Figure 3b) [79]. More importantly, the scratches and microcracks of the conductive composite layer can be healed in $3 \mathrm{~min}$ at $80^{\circ} \mathrm{C}$; this performance greatly extends its lifetime (Figure 3c) [79]. Graphene platelets/PDMS strain sensors allow the structure to be repeatedly bent and stretched with superior physical robustness and cyclability. It exhibits high durability that responds to cyclic loading with remarkable stability even after thousands of cycles at $5 \%$ and $10 \%$ strain (Figure 3d,e) [80]. So far, researchers have paid more attention to the mechanical robustness of wearable strain sensors, while few studies have focused on the feasibility in hostile environments, which needs further study.
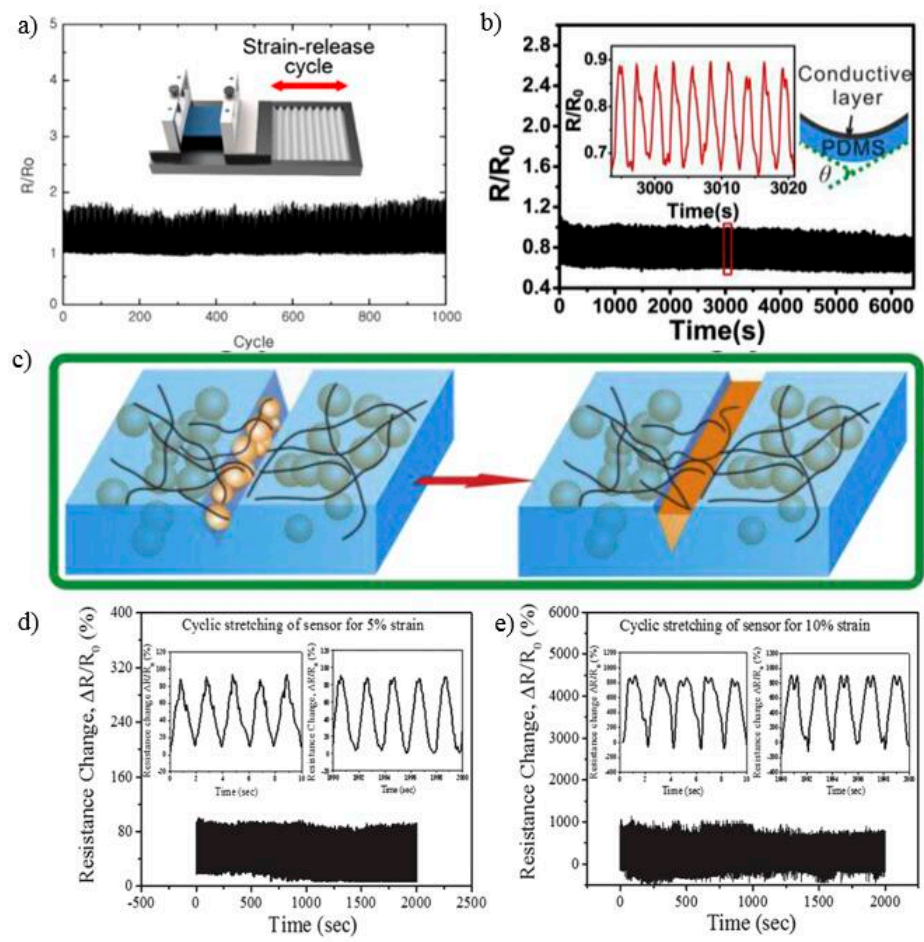

Figure 3. (a) Resistance change of silver nanowire (AgNW)/PDMS percolation network strain sensor under a long-term cyclic strain response test (103 cycles). Reprinted with permission from [54] Copyright American Chemical Society, 2015; (b) Response of the two-layered flexible strain sensors to the bending cycles during more than 2400 bending cycles. Reprinted with permission from [79], Copyright Elsevier, 2017; (c) Healing procedure of the two-layered flexible strain sensors with microcracks. Reprinted with permission [79], Copyright Elsevier, 2017; (d,e) Characterization of graphene platelets/PDMS strain sensors shows high durability over 1000 cycles for different strains (5\% and 10\%). Reprinted with permission from [80], Copyright John Wiley and Sons, 2016.

In summary, the advantages of PDMS-based flexible resistive strain sensors are obvious, some of them are compared and summarized in Table 1 . They have high tensile properties and good stability, particularly suitable for wearable fields such as human entertainment technology, human-machine interface, personal healthcare, and sports performance monitoring, etc. However, because deformation-uneven microstructure heavily impacts the input-output response, there is always a trade-off relationship between 
high excellent linearity and high sensitivity. Therefore, developing a better performance strain sensor is still a challenge.

Table 1. Comparison of polydimethylsiloxane (PDMS)-based flexible strain sensors.

\begin{tabular}{|c|c|c|c|c|c|}
\hline \multirow{2}{*}{ Materials } & \multirow{2}{*}{ Fabrication Methods } & \multicolumn{3}{|c|}{ Performances } & \multirow{2}{*}{ Applications } \\
\hline & & Stretching & GFs & Linearity & \\
\hline EGaIn-PDMS [67] & Printing technology & $350 \%$ & $1.6-3.2$ & Partly linear & $\begin{array}{l}\text { Wearable devices; soft robots; } \\
\text { electronic skin }\end{array}$ \\
\hline AgNW-PDMS [68] & Micromolding method & $70 \%$ & $2-14$ & Partly linear & Motion detection ; virtual reality \\
\hline CNT-CB-PDMS [58] & Micromolding method & $22.6 \%$ & 29 & Nonlinear & $\begin{array}{l}\text { Human-machine interfaces; } \\
\text { wound monitoring }\end{array}$ \\
\hline CB-PDMS [75] & Liquid phase mixing & $10 \%$ & 5.5 & linear & Real-time monitoring \\
\hline Graphene-PDMS [71] & Coating techniques & $7.1 \%$ & $2.4-14$ & $\begin{array}{l}\text { Two linear } \\
\text { regions }\end{array}$ & $\begin{array}{c}\text { Wearable devices; } \\
\text { multi-parameter monitoring }\end{array}$ \\
\hline GWFs-PDMS [72] & Coating techniques & $30 \%$ & $\sim 10^{6}$ & Nonlinear & $\begin{array}{c}\text { Displays; robotics; fatigue } \\
\text { detection; advanced therapies; } \\
\text { in vitro diagnostic }\end{array}$ \\
\hline MWCNTs-PDMS [69] & Liquid phase mixing & $45 \%$ & 1.2 & Nonlinear & Biomedical industry \\
\hline MWNT-GNPs-PDMS [65] & Printing technology & $40 \%$ & $>100$ & Partly linear & $\begin{array}{l}\text { Health diagnostics } \\
\text { and monitoring }\end{array}$ \\
\hline AgNP-PDMS [47] & Printing technology & $20 \%$ & $4.7-12.5$ & $\begin{array}{l}\text { Two linear } \\
\text { regions }\end{array}$ & $\begin{array}{c}\text { Human motion monitoring; force } \\
\text { and pressure } \\
\text { measurement devices }\end{array}$ \\
\hline CNT-PDMS [59] & Printing technology & $100 \%$ & $2 \times 10^{4}$ & Nonlinear & $\begin{array}{l}\text { Measuring joints motions } \\
\text { in real-time }\end{array}$ \\
\hline GO-PDMS [61] & Filtration method & $\begin{array}{l}\text { Bending radii } \\
(5 \mathrm{~cm}-2.5 \mathrm{~cm})\end{array}$ & - & Nonlinear & $\begin{array}{c}\text { Measuring the bending angle of } \\
\text { a joints }\end{array}$ \\
\hline $\begin{array}{l}\text { Graphene- } \\
\text { nanocellulose-PDMS [63] }\end{array}$ & Filtration method & $100 \%$ & 7.1 & Partly linear & Human interactive \\
\hline Ti/Au-PDMS [53] & Micromolding method & - & - & Nonlinear & $\begin{array}{l}\text { Measuring drug-induced changes } \\
\text { in cells }\end{array}$ \\
\hline GnPs-PDMS [80] & Micromolding method & $<10 \%$ & $27.7-164.5$ & Nonlinear & $\begin{array}{l}\text { Health monitoring; electronic } \\
\text { skin; human-machine interface }\end{array}$ \\
\hline
\end{tabular}

\section{Applications}

There are numerous potential applications for PDMS-based flexible resistive strain sensors such as virtual reality and entertainment technology, human-machine interfaces, personal healthcare, and sports performance monitoring, etc. These kinds of strain sensors can be attached to clothing or directly laminated on the human skin because PDMS flexible substrates are both stretchable and biocompatible. An avatar in the computer virtual environment was successfully controlled by using smart gloves integrated with AgNWs/PDMS sandwich-structured strain sensors. Each sensor was fixedly attached to the back of each finger with the fingers bending, leading to the bending of the avatar finger (Figure 4a) [68]. Meanwhile, an intelligent glove was fabricated with nine carbon particle/PDMS sensors and wearable electronic module; the nine-digit binary keys were based on the bending/unbending states of the nine sensors. These keys were associated with 26 letters of the American Sign Language (ASL) alphabet. The corresponding gestures of the glove successfully translated all letters into text displayable on a computer screen (Figure 4b) [81]. Human-machine interface is another potential application. The signals from PDMS-based flexible resistive strain sensors can be utilized to detect or actuate smart robots. The graphene/PDMS-based sensors were directly fixed across the movable joints of a robot to detect elbow rotation, waist side-bending, and stepping (Figure 4c) [82]; those strain sensors successfully accomplished the real-time movement detection during the robot dancing "Gangnam Style". As demonstrated in Figure 4d, the carbon paper (CP) /PDMS strain sensor was employed to control the movement of a mobile robotic platform. The forward and backward motion of the moving robot depends on whether the CP/PDMS strain sensor is being stretched or relaxed [83]. The CP/PDMS strain sensor shows excellent stability when used in a man-robot interaction scenario. Personal healthcare monitoring is promising to deal with poor medical services. A wearable strain sensor has been assembled by graphene woven fabrics (GWFs) 
on polymer and measures physiological signals [72]. Diagnosis and therapy of movement disorders have been achieved using silicon nanomembrane ( $\mathrm{Si} N \mathrm{NM}$ ) strain sensors where the monitored data was stored in integrated memory devices gold nanoparticle (Au NP) RRAMs and drug delivery from mesoporous-silica (m-silica) nanoparticles proceeded transdermally [14].

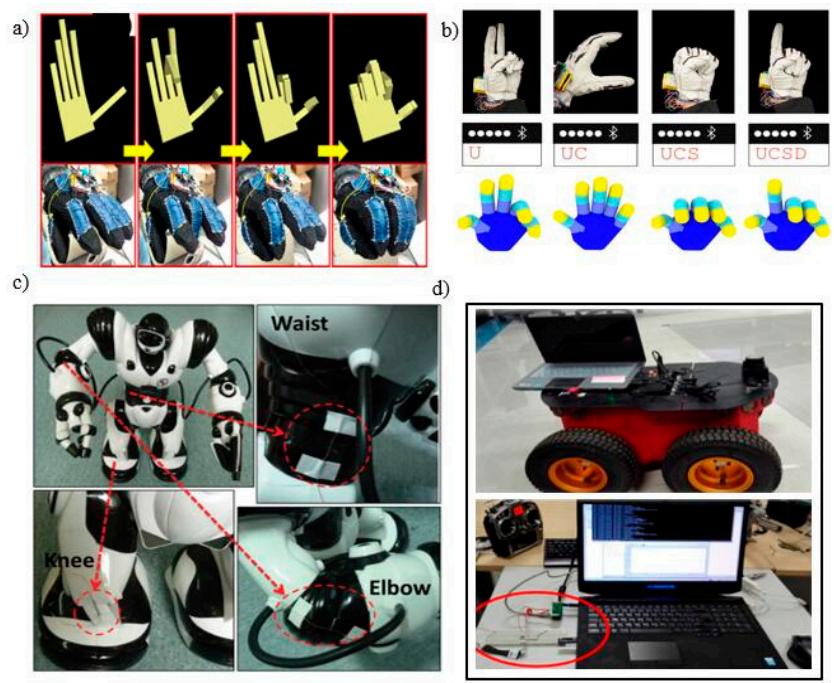

Figure 4. (a) A smart glove system made of five strain sensors control the avatar fingers in the virtual environment. Reprinted with permission from [68], Copyright American Chemical Society, 2014; (b) A low-cost system for decoding and transmitting human hand gestures by generating digit binary key based on the on/off states of the strain sensors. Reprinted with permission from [81], Copyright PLOS, 2017; (c) The robot wore graphene/PDMS-based sensors at movable joints using adhesive tape to detect various kinds of motion. Reprinted with permission from [82], Copyright John Wiley and Sons, 2015; (d) A mobile robotic platform controlled by the carbon paper (CP)/PDMS strain sensor. Reprinted with permission from [83], Copyright American Chemical Society, 2016.

Some weak human motions including phonation, blinking, breathing, and pulses have been easily detected because GWFs-PDMS-tape sensors are high sensitivity and have superior physical robustness and reversible extensibility. As shown in Figure 5a, all test data of the breathing rate during rest and exercise states were within the normal range of a healthy adult. Nanoscale crack junctions of Pt film-based sensors were applied for medical physiological signal collection (Figure 5b) [78]. The flexible strain sensor was attached to a human neck for speech pattern recognition; the accuracy of simple word recognition was approximately $97.5 \%$ even in a noisy environment. Another application of the nanoscale crack sensor was heart rate measuring; the diastolic and systolic movements of the heart were successfully monitored. Wearable strain sensors can also function in sports performance monitoring. A proof-of-concept device was demonstrated composed of five U-shaped graphene strain sensors implanted on a common glove to detect the bending and stretching of fingers. The data glove could measure the deformation of fingers in the strain range of $35-45 \%$ (Figure 5c) [63]. A large single-walled carbon nanotube (SWCNT)/PDMS-based strain sensor was assembled in a commercial stocking over the knee joint, and could detect and distinguish every movement of the knee including bending, marching, squatting, and jumping(Figure 5d) [46]. 


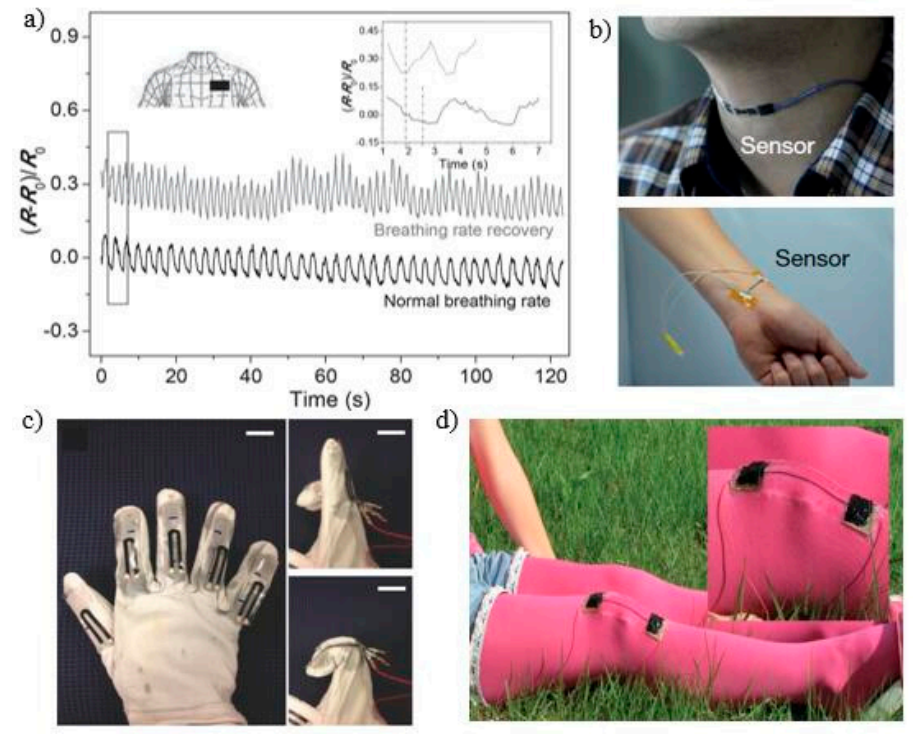

Figure 5. (a) The graphene woven fabric (GWF)/PDMS-type sensor's relative change of resistance of the respiratory in the rest and exercise states, respectively. Reprinted with permission from [72], Copyright John Wiley and Sons, 2014; (b) The nanoscale crack sensor attached to skin recording human speech and pulse measurement. Reprinted by permission from [78], Copyright Springer Nature, 2014; (c) Data glove with five implanted sensors detected finger moving. Reprinted with permission from [63], Copyright John Wiley and Sons, 2013; (d) A wearable carbon nanotube strain sensor integrated into a stocking to detect knee motion. Reprinted with permission from [46], Copyright Springer Nature, 2011.

\section{Conclusions and Outlook}

We have presented a comprehensive review of the recent technological advances of PDMS-based flexible resistive strain sensors. We introduced substrate and active materials, several fabrication methods, performance parameters, and their applications. It leads us to believe that flexible strain sensors can potentially function in many applications, such as virtual reality and entertainment technology, human-machine interfaces, personal healthcare, and sports performance monitoring. Wearable intelligent electronic systems will play an increasingly critical role in our daily life. Nevertheless, the wearable sensors in practical applications are still facing huge challenges. At present, most of the strain sensors have sole functions and can only monitor deformation in one direction because they are not capable of decoupling more than one type of stimuli at a time. Strain sensors measuring decoupled strains in multi-direction or multi-plane deformations have not been successfully fabricated yet, even though many strain- and pressure-sensitive sensors have been reported. Besides, strain sensors with relatively high modulus materials and weak adhesion structures make them wearing uncomfortable and lead them to easily slide off their particular position. Therefore, they cannot be reliably integrated with the human body for real-time effective deformation measurements. Moreover, packaging flexible sensors with other components has received less attention. There are still some problems including the followings: the electronic circuit is rigid and the size is not small, making it impractical to integrate with soft sensors; the communication module electricity consumption is huge even using low-power miniaturized Bluetooth technologies; the current thin-film batteries lack sufficient capacity and recharging is complex and time-consuming, etc. To solve these problems, in addition to selecting or developing functional materials, more efficient methods and optimization of devices are needed to be explored for improving the sensing performance.

Acknowledgments: This research was partially supported by Shenzhen Fundamental Research Project (JCYJ20170307165039508), the National Natural Science Foundation of China (NSFC: U1713219), Key Deployment Project of Chinese Academy of Sciences (Grant No. KFZD-SW-214), and SIAT Innovation Program for Excellent Young Researchers. (Grant No. 2016053). 
Author Contributions: H.L. and L.W. proposed the idea; H.L. and J.C. conceived and designed the structure of this review; J.C., J.Z., Q.G., J.Z., J.Z., O.M.O., and H.L. contributed the materials, figures, and tables for this review; H.L. and J.C. wrote the paper.

Conflicts of Interest: The authors declare no conflict of interest.

\section{References}

1. Choi, M.K.; Park, I.; Kim, D.C.; Joh, E.; Park, O.K.; Kim, J.; Kim, M.; Choi, C.; Yang, J.; Cho, K.W. Thermally controlled, patterned graphene transfer printing for transparent and wearable electronic/optoelectronic system. Adv. Funct. Mater. 2016, 25, 7109-7118. [CrossRef]

2. Tee, B.C.; Chortos, A.; Berndt, A.; Nguyen, A.K.; Tom, A.; Mcguire, A.; Lin, Z.C.; Tien, K.; Bae, W.G.; Wang, H. A skin-inspired organic digital mechanoreceptor. Science 2015, 350, 313-316. [CrossRef] [PubMed]

3. Park, Y.L.; Chen, B.R.; Wood, R.J. Design and fabrication of soft artificial skin using embedded microchannels and liquid conductors. IEEE Sens. J. 2012, 12, 2711-2718. [CrossRef]

4. Yan, C.; Wang, J.; Kang, W.; Cui, M.; Wang, X.; Foo, C.Y.; Chee, K.J.; Lee, P.S. Highly stretchable piezoresistive graphene-nanocellulose nanopaper for strain sensors. Adv. Mater. 2014, 26, 2022-2027. [CrossRef] [PubMed]

5. Ponce Wong, R.D.; Posner, J.D.; Santos, V.J. Flexible microfluidic normal force sensor skin for tactile feedback. Sens. Actuators A Phys. 2012, 179, 62-69. [CrossRef]

6. Wang, X.; Gu, Y.; Xiong, Z.; Cui, Z.; Zhang, T. Electronic skin: Silk-molded flexible, ultrasensitive, and highly stable electronic skin for monitoring human physiological signals (Adv. Mater. 9/2014). Adv. Mater. 2014, $26,1309$. [CrossRef]

7. Ossig, C.; Antonini, A.; Buhmann, C.; Classen, J.; Csoti, I.; Falkenburger, B.; Schwarz, M.; Winkler, J.; Storch, A. Wearable sensor-based objective assessment of motor symptoms in parkinson's disease. J. Neural Transm. 2016, 123, 57-64. [CrossRef] [PubMed]

8. Son, D.; Koo, J.H.; Song, J.-K.; Kim, J.; Lee, M.; Shim, H.J.; Park, M.; Lee, M.; Kim, J.H.; Kim, D.-H. Stretchable carbon nanotube charge-trap floating-gate memory and logic devices for wearable electronics. ACS Nano 2015, 9, 5585-5593. [CrossRef] [PubMed]

9. Son, D.; Chae, S.I.; Kim, M.; Choi, M.K.; Yang, J.; Park, K.; Kale, V.S.; Koo, J.H.; Choi, C.; Lee, M. Colloidal synthesis of uniform-sized molybdenum disulfide nanosheets for wafer-scale flexible nonvolatile memory. Adv. Mater. 2016, 28, 9326-9332. [CrossRef] [PubMed]

10. Tien, N.T.; Jeon, S.; Kim, D.I.; Trung, T.Q.; Jang, M.; Hwang, B.U.; Byun, K.E.; Bae, J.; Lee, E.; Tok, J.B. A flexible bimodal sensor array for simultaneous sensing of pressure and temperature. Adv. Mater. 2014, 26, 796-804. [CrossRef] [PubMed]

11. Gao, W.; Emaminejad, S.; Nyein, H.Y.; Challa, S.; Chen, K.; Peck, A.; Fahad, H.M.; Ota, H.; Shiraki, H.; Kiriya, D. Fully integrated wearable sensor arrays for multiplexed in situ perspiration analysis. Nature 2016, 529, 509-514. [CrossRef] [PubMed]

12. Zhang, D.; Matsuoka, Y.; Kong, W.; Imtiaz, U.; Bartolomeo, L.; Cosentino, S.; Zecca, M.; Sessa, S.; Ishii, H.; Takanishi, A. Development of New Muscle Contraction Sensor to Replace Semg for Using in Muscles Analysis Fields. In Proceedings of the 2014 36th Annual International Conference of the IEEE Engineering in Medicine and Biology Society, Chicago, IL, USA, 26-30 August 2014; pp. 6945-6948.

13. Gao, L.; Dong, D.; He, J.; Qiao, K.; Cao, F.; Li, M.; Liu, H.; Cheng, Y.; Tang, J.; Song, H. Wearable and sensitive heart-rate detectors based on pbs quantum dot and multiwalled carbon nanotube blend film. Appl. Phys. Lett. 2014, 105, 838-899. [CrossRef]

14. Son, D.; Lee, J.; Qiao, S.; Ghaffari, R.; Kim, J.; Lee, J.E.; Song, C.; Kim, S.J.; Lee, D.J.; Jun, S.W. Multifunctional wearable devices for diagnosis and therapy of movement disorders. Nat. Nanotechnol. 2014, 9, 397-404. [CrossRef] [PubMed]

15. Tadano, S.; Takeda, R.; Miyagawa, H. Three dimensional gait analysis using wearable acceleration and gyro sensors based on quaternion calculations. Sensors 2013, 13, 9321-9343. [CrossRef] [PubMed]

16. Yao, S.; Zhu, Y. Wearable multifunctional sensors using printed stretchable conductors made of silver nanowires. Nanoscale 2014, 6, 2345-2352. [CrossRef] [PubMed]

17. Grosshauser, T.; Bläsing, B.; Spieth, C.; Hermann, T. Wearable sensor-based real-time sonification of motion and foot pressure in dance teaching and training. J. Audio Eng. Soc. 2012, 60, 580-589. 
18. Tao, W.; Liu, T.; Zheng, R.; Feng, H. Gait analysis using wearable sensors. Sensors 2012, 12, $2255-2283$. [CrossRef] [PubMed]

19. Takeda, R.; Lisco, G.; Fujisawa, T.; Gastaldi, L.; Tohyama, H.; Tadano, S. Drift removal for improving the accuracy of gait parameters using wearable sensor systems. Sensors 2014, 14, 23230-23247. [CrossRef] [PubMed]

20. Qian, W.; Zhao, C.L.; He, S.; Dong, X.; Zhang, S.; Zhang, Z.; Jin, S.; Guo, J.; Wei, H. High-sensitivity temperature sensor based on an alcohol-filled photonic crystal fiber loop mirror. Opt. Lett. 2011, 36, 1548-1550. [CrossRef] [PubMed]

21. Yang, J.; Wei, D.; Tang, L.; Song, X.; Luo, W.; Chu, J.; Gao, T.; Shi, H.; Du, C. Wearable temperature sensor based on graphene nanowalls. RSC Adv. 2015, 5, 25609-25615. [CrossRef]

22. Mahadeva, S.K.; Yun, S.; Kim, J. Flexible humidity and temperature sensor based on cellulose-polypyrrole nanocomposite. Sens. Actuators A Phys. 2011, 165, 194-199. [CrossRef]

23. Lin, Q.; Li, Y.; Yang, M. Polyaniline nanofiber humidity sensor prepared by electrospinning. Sens. Actuators B Chem. 2012, 161, 967-972. [CrossRef]

24. Yu, K.; Bo, Z.; Lu, G.; Mao, S.; Cui, S.; Zhu, Y.; Chen, X.; Ruoff, R.S.; Chen, J. Growth of carbon nanowalls at atmospheric pressure for one-step gas sensor fabrication. Nanoscale Res. Lett. 2011, 6, 202. [CrossRef] [PubMed]

25. Choi, D.Y.; Kim, M.H.; Oh, Y.S.; Jung, S.H.; Jung, J.H.; Sung, H.J.; Lee, H.W.; Lee, H.M. Highly stretchable, hysteresis-free ionic liquid-based strain sensor for precise human motion monitoring. ACS Appl. Mater. Interfaces 2017, 9, 1770-1780. [CrossRef] [PubMed]

26. Zhang, M.; Wang, C.; Wang, H.; Jian, M.; Hao, X.; Zhang, Y. Carbonized cotton fabric for high-performance wearable strain sensors. Adv. Funct. Mater. 2016, 27. [CrossRef]

27. Li, Y.-Q.; Huang, P.; Zhu, W.-B.; Fu, S.-Y.; Hu, N.; Liao, K. Flexible wire-shaped strain sensor from cotton thread for human health and motion detection. Sci. Rep. 2017, 7, 45013. [CrossRef] [PubMed]

28. Zheng, Y.; Li, Y.; Dai, K.; Wang, Y.; Zheng, G.; Liu, C.; Shen, C. A highly stretchable and stable strain sensor based on hybrid carbon nanofillers/polydimethylsiloxane conductive composites for large human motions monitoring. Compos. Sci. Technol. 2018. [CrossRef]

29. Xiao, X.; Yuan, L.; Zhong, J.; Ding, T.; Liu, Y.; Cai, Z.; Rong, Y.; Han, H.; Zhou, J.; Wang, Z.L. High-strain sensors based on zno nanowire/polystyrene hybridized flexible films. Adv. Mater. 2011, 23, 5440-5444. [CrossRef] [PubMed]

30. Shi, J.; Li, X.; Cheng, H.; Liu, Z.; Zhao, L.; Yang, T.; Dai, Z.; Cheng, Z.; Shi, E.; Yang, L. Graphene reinforced carbon nanotube networks for wearable strain sensors. Adv. Funct. Mater. 2016, 26, 2078-2084. [CrossRef]

31. Wang, C.; Xia, K.; Jian, M.; Wang, H.; Zhang, M.; Zhang, Y. Carbonized silk georgette as an ultrasensitive wearable strain sensor for full-range human activity monitoring. J. Mater. Chem. C 2017, 5, 7604-7611. [CrossRef]

32. Kim, D.-H.; Lu, N.; Ma, R.; Kim, Y.-S.; Kim, R.-H.; Wang, S.; Wu, J.; Won, S.M.; Tao, H.; Islam, A. Epidermal electronics. Science 2011, 333, 838-843. [CrossRef] [PubMed]

33. Guo, X.; Huang, Y.; Cai, X.; Liu, C.; Liu, P. Capacitive wearable tactile sensor based on smart textile substrate with carbon black / silicone rubber composite dielectric. Meas. Sci. Technol. 2016, 27, 045105. [CrossRef]

34. Bansal, A.K.; Hou, S.; Kulyk, O.; Bowman, E.M.; Samuel, I.D.W. Wearable organic optoelectronic sensors for medicine. Adv. Mater. 2015, 27, 7638-7644. [CrossRef] [PubMed]

35. Alluri, N.R.; Selvarajan, S.; Chandrasekhar, A.; Saravanakumar, B.; Ji, H.J.; Kim, S.J. Piezoelectric batio 3 /alginate spherical composite beads for energy harvesting and self-powered wearable flexion sensor. Compos. Sci. Technol. 2017, 142, 65-78. [CrossRef]

36. Wang, C.; Li, X.; Gao, E.; Jian, M.; Xia, K.; Wang, Q.; Xu, Z.; Ren, T.; Zhang, Y. Carbonized silk fabric for ultrastretchable, highly sensitive, and wearable strain sensors. Adv. Mater. 2016, 28, 6640-6648. [CrossRef] [PubMed]

37. Liao, X.; Zhang, Z.; Kang, Z.; Gao, F.; Liao, Q.; Zhang, Y. Ultrasensitive and stretchable resistive strain sensors designed for wearable electronics. Mater. Horiz. 2017, 4, 502-510. [CrossRef]

38. Juárez-Moreno, J.A.; Ávila-Ortega, A.; Oliva, A.I.; Avilés, F.; Cauich-Rodríguez, J.V. Effect of wettability and surface roughness on the adhesion properties of collagen on pdms films treated by capacitively coupled oxygen plasma. Appl. Surf. Sci. 2015, 349, 763-773. [CrossRef] 
39. Chen, D.; Chen, F.; Hu, X.; Zhang, H.; Yin, X.; Zhou, Y. Thermal stability, mechanical and optical properties of novel addition cured pdms composites with nano-silica sol and mq silicone resin. Compos. Sci. Technol. 2015, 117, 307-314. [CrossRef]

40. Johnston, I.D.; Mccluskey, D.K.; Tan, C.K.L.; Tracey, M.C. Mechanical characterization of bulk sylgard 184 for microfluidics and microengineering. J. Micromech. Microeng. 2014, 24, 10-11. [CrossRef]

41. Fan, X.; Jia, C.; Yang, J.; Li, G.; Mao, H.; Jin, Q.; Zhao, J. A microfluidic chip integrated with a high-density pdms-based microfiltration membrane for rapid isolation and detection of circulating tumor cells. Biosens. Bioelectron. 2015, 71, 380-386. [CrossRef] [PubMed]

42. Singh, S.; Kumar, N.; George, D.; Sen, A.K. Analytical modeling, simulations and experimental studies of a pzt actuated planar valveless pdms micropump. Sens. Actuators A Phys. 2015, 225, 81-94. [CrossRef]

43. Wang, X.; Gu, Y.; Xiong, Z.; Cui, Z.; Zhang, T. Silk-molded flexible, ultrasensitive, and highly stable electronic skin for monitoring human physiological signals. Adv. Mater. 2014, 26, 1336-1342. [CrossRef] [PubMed]

44. Kakati, A.; Das, S. Nano-structured gold strain gauge arrays on pdms for highly sensitive nems pressure sensor skin. In Proceedings of the 2017 IEEE 12th International Conference on Nano/Micro Engineered and Molecular Systems (NEMS), Los Angeles, CA, USA, 9-12 April 2017; IEEE: Piscataway, NJ, USA, 2017; pp. 611-614.

45. Shin, U.H.; Jeong, D.W.; Park, S.M.; Kim, S.H.; Lee, H.W.; Kim, J.M. Highly stretchable conductors and piezocapacitive strain gauges based on simple contact-transfer patterning of carbon nanotube forests. Carbon 2014, 80, 396-404. [CrossRef]

46. Yamada, T.; Hayamizu, Y.; Yamamoto, Y.; Yomogida, Y.; Izadi-Najafabadi, A.; Futaba, D.N.; Hata, K. A stretchable carbon nanotube strain sensor for human-motion detection. Nat. Nanotechnol. 2011, 6, $296-301$. [CrossRef] [PubMed]

47. Lee, J.H.; Yang, D.; Kim, S.; Park, I. Stretchable strain sensor based on metal nanoparticle thin film for human motion detection \& flexible pressure sensing devices. Nanoscale 2014, 6, 11932. [CrossRef] [PubMed]

48. Yaqoob, U.; Phan, D.T.; Uddin, A.S.M.I.; Chung, G.S. Highly flexible room temperature no 2 sensor based on mwcnts-wo 3 nanoparticles hybrid on a pet substrate. Sens. Actuators B Chem. 2015, 221, 760-768. [CrossRef]

49. Kim, J.Y.; Ji, S.; Jung, S.; Ryu, B.H.; Kim, H.S.; Lee, S.S.; Choi, Y.; Jeong, S. 3D printable composite dough for stretchable, ultrasensitive and body-patchable strain sensors. Nanoscale 2017, 9, 11035-11046. [CrossRef] [PubMed]

50. Olichwer, N.; Leib, E.W.; Halfar, A.H.; Petrov, A.; Vossmeyer, T. Cross-linked gold nanoparticles on polyethylene: Resistive responses to tensile strain and vapors. ACS Appl. Mater. Interfaces 2012, 4, 6151-6161. [CrossRef] [PubMed]

51. Wang, J.; Jiu, J.; Nogi, M.; Sugahara, T.; Nagao, S.; Koga, H.; He, P.; Suganuma, K. A highly sensitive and flexible pressure sensor with electrodes and elastomeric interlayer containing silver nanowires. Nanoscale 2015, 7, 2926-2932. [CrossRef] [PubMed]

52. Yu, X.G.; Li, Y.Q.; Zhu, W.B.; Huang, P.; Wang, T.T.; Hu, N.; Fu, S.Y. A wearable strain sensor based on a carbonized nano-sponge/silicone composite for human motion detection. Nanoscale 2017, 9, 6680-6685. [CrossRef] [PubMed]

53. Kim, D.S.; Jeong, Y.J.; Lee, B.K.; Shanmugasundaram, A.; Lee, D.W. Piezoresistive sensor-integrated pdms cantilever: A new class of device for measuring the drug-induced changes in the mechanical activity of cardiomyocytes. Sens. Actuators B Chem. 2017, 240, 566-572. [CrossRef]

54. Kim, K.K.; Hong, S.; Cho, H.M.; Lee, J.; Suh, Y.D.; Ham, J.; Ko, S.H. Highly sensitive and stretchable multidimensional strain sensor with prestrained anisotropic metal nanowire percolation networks. Nano Lett. 2015, 15, 5240-5247. [CrossRef] [PubMed]

55. Eom, H.; Lee, J.; Pichitpajongkit, A.; Amjadi, M.; Jeong, J.H.; Lee, E.; Lee, J.Y.; Park, I. Ag@Ni core-shell nanowire network for robust transparent electrodes against oxidation and sulfurization. Small 2014, 10, 4171-4181. [CrossRef] [PubMed]

56. Zhang, S.; Zhang, H.; Yao, G.; Liao, F.; Gao, M.; Huang, Z.; Li, K.; Lin, Y. Highly stretchable, sensitive, and flexible strain sensors based on silver nanoparticles/carbon nanotubes composites. J. Alloys Compd. 2015, 652, 48-54. [CrossRef]

57. Coskun, M.B.; Akbari, A.; Lai, D.; Neild, A.; Majumder, M.; Alan, T. Ultrasensitive strain sensor produced by direct patterning of liquid crystals of graphene oxide on a flexible substrate. ACS Appl. Mater. Interfaces 2016, 8, 22501-22505. [CrossRef] [PubMed] 
58. Lu, N.; Lu, C.; Yang, S.; Rogers, J. Highly Sensitive Skin-Mountable Strain gauges Based Entirely on Elastomers. Adv. Funct. Mater. 2012, 22, 4044-4050. [CrossRef]

59. Rahimi, R.; Ochoa, M.; Yu, W.; Ziaie, B. Highly stretchable and sensitive unidirectional strain sensor via laser carbonization. ACS Appl. Mater. Interfaces 2015, 7, 4463-4470. [CrossRef] [PubMed]

60. Amjadi, M.; Kyung, K.U.; Park, I.; Sitti, M. Stretchable, skin-mountable, and wearable strain sensors and their potential applications: A review. Adv. Funct. Mater. 2016, 26, 1678-1698. [CrossRef]

61. Ren, J.; Wang, C.; Zhang, X.; Tian, C.; Chen, K.; Yin, Y.; Torrisi, F. Environmentally-friendly conductive cotton fabric as flexible strain sensor based on hot press reduced graphene oxide. Carbon 2017, 111, 622-630. [CrossRef]

62. Bo, M.L.; Loh, K.J.; Yang, Y.S. Carbon nanotube thin film strain sensor models assembled using nano- and micro-scale imaging. Comput. Mech. 2017, 60, 39-49.

63. Yan, C.; Wang, J.; Kang, W.; Cui, M.; Wang, X.; Foo, C.Y.; Chee, K.J.; Lee, P.S. Graphene: Highly stretchable piezoresistive graphene-nanocellulose nanopaper for strain sensors (Adv. Mater. 13/2014). Adv. Mater. 2014, 26, 1950. [CrossRef]

64. Lee, H.; Seong, B.; Moon, H.; Byun, D. Directly printed stretchable strain sensor based on ring and diamond shaped silver nanowire electrodes. RSC Adv. 2015, 5, 28379-28384. [CrossRef]

65. Lee, C.; Jug, L.; Meng, E. Erratum: "High strain biocompatible polydimethylsiloxane-based conductive graphene and multiwalled carbon nanotube nanocomposite strain sensors" [Appl. Phys. Lett. 102(18), 183511 (2013)]. Appl. Phys. Lett. 2013, 103. [CrossRef]

66. Boley, J.W.; White, E.L.; Chiu, G.T.C.; Kramer, R.K. Direct writing of gallium-indium alloy for stretchable electronics. Adv. Funct. Mater. 2014, 24, 3501-3507. [CrossRef]

67. Yan, H.; Chen, Y.; Deng, Y.; Zhang, L.; Hong, X.; Lau, W.; Mei, J.; Hui, D.; Yan, H.; Liu, Y. Coaxial printing method for directly writing stretchable cable as strain sensor. Appl. Phys. Lett. 2016, 109. [CrossRef]

68. Amjadi, M.; Pichitpajongkit, A.; Lee, S.; Ryu, S.; Park, I. Highly stretchable and sensitive strain sensor based on silver nanowire-elastomer nanocomposite. ACS Nano 2014, 8, 5154-5163. [CrossRef] [PubMed]

69. Liu, C.X.; Choi, J.W. Analyzing resistance response of embedded pdms and carbon nanotubes composite under tensile strain. Microelectron. Eng. 2014, 117, 1-7. [CrossRef]

70. Choong, C.L.; Shim, M.B.; Lee, B.S.; Jeon, S.; Ko, D.S.; Kang, T.H.; Bae, J.; Lee, S.H.; Byun, K.E.; Im, J. Highly stretchable resistive pressure sensors using a conductive elastomeric composite on a micropyramid array. Adv. Mater. 2014, 26, 3451-3458. [CrossRef] [PubMed]

71. Bae, S.H.; Lee, Y.; Sharma, B.K.; Lee, H.J.; Kim, J.H.; Ahn, J.H. Graphene-based transparent strain sensor. Carbon 2013, 51, 236-242. [CrossRef]

72. Wang, Y.; Wang, L.; Yang, T.; Li, X.; Zang, X.; Zhu, M.; Wang, K.; Wu, D.; Zhu, H. Wearable and highly sensitive graphene strain sensors for human motion monitoring. Adv. Funct. Mater. 2014, 24, 4666-4670. [CrossRef]

73. Xiao, L.; Zhang, R.; Yu, W.; Wang, K.; Wei, J.; Wu, D.; Cao, A.; Li, Z.; Yao, C.; Zheng, Q. Stretchable and highly sensitive graphene-on-polymer strain sensors. Sci. Rep. 2012, 2, 870. [CrossRef]

74. Wang, B.; Lee, B.K.; Kwak, M.J.; Lee, D.W. Graphene/polydimethylsiloxane nanocomposite strain sensor. Rev. Sci. Instrum. 2013, 84, 105005. [CrossRef] [PubMed]

75. Kong, J.H.; Jang, N.S.; Kim, S.H.; Kim, J.M. Simple and rapid micropatterning of conductive carbon composites and its application to elastic strain sensors. Carbon 2014, 77, 199-207. [CrossRef]

76. Xu, F.; Zhu, Y. Highly conductive and stretchable silver nanowire conductors. Adv. Mater. 2012, 24, 5117-5122. [CrossRef] [PubMed]

77. Park, H.; Kim, D.S.; Hong, S.Y.; Kim, C.; Yun, J.Y.; Oh, S.Y.; Jin, S.W.; Jeong, Y.R.; Kim, G.T.; Ha, J.S. A skin-integrated transparent and stretchable strain sensor with interactive color-changing electrochromic displays. Nanoscale 2017, 9, 7631-7640. [CrossRef] [PubMed]

78. Kang, D.; Pikhitsa, P.V.; Yong, W.C.; Lee, C.; Shin, S.S.; Piao, L.; Park, B.; Suh, K.Y.; Kim, T.; Choi, M. Ultrasensitive mechanical crack-based sensor inspired by the spider sensory system. Nature 2014, 516, 222-226. [CrossRef] [PubMed]

79. Liu, S.; Lin, Y.; Wei, Y.; Chen, S.; Zhu, J.; Liu, L. A high performance self-healing strain sensor with synergetic networks of poly(E-caprolactone) microspheres, graphene and silver nanowires. Compos. Sci. Technol. 2017, 146, 110-118. [CrossRef] 
80. Shi, G.; Zhao, Z.; Pai, J.H.; Lee, I.; Zhang, L.; Stevenson, C.; Ishara, K.; Zhang, R.; Zhu, H.; Ma, J. Highly sensitive, wearable, durable strain sensors and stretchable conductors using graphene/silicon rubber composites. Adv. Funct. Mater. 2016, 26, 7614-7625. [CrossRef]

81. O'Connor, T.F.; Fach, M.E.; Miller, R.; Root, S.E.; Mercier, P.P.; Lipomi, D.J. The language of glove: Wireless gesture decoder with low-power and stretchable hybrid electronics. PLoS ONE 2017, 12, e0179766. [CrossRef] [PubMed]

82. Cheng, Y.; Wang, R.; Sun, J.; Gao, L. A stretchable and highly sensitive graphene-based fiber for sensing tensile strain, bending, and torsion. Adv. Mater. 2015, 27, 7365-7371. [CrossRef] [PubMed]

83. Li, Y.; Samad, Y.A.; Taha, T.; Cai, G.; Fu, S.Y.; Liao, K. Highly flexible strain sensor from tissue paper for wearable electronics. ACS Sustain. Chem. Eng. 2016, 4, 4288-4295. [CrossRef]

2018 by the authors. Licensee MDPI, Basel, Switzerland. This article is an open access article distributed under the terms and conditions of the Creative Commons Attribution (CC BY) license (http://creativecommons.org/licenses/by/4.0/). 